\title{
Parallel end-butt coupling for optical integrated circuits
}

\author{
R. G. Hunsperger, A. Yariv, and A. Lee
}

\begin{abstract}
The method of parallel end-butt coupling has been used to couple $\mathrm{GaAs}$ laser diodes to $\mathrm{Ta}_{2} \mathrm{O}_{5}$ thin film waveguides. Theoretical caluclations predict that a coupling efficiency into the lowest order waveguide mode of $90 \%$ is achievable if the thicknesses of the waveguide $\left(t_{g}\right)$ and the laser light emitting layer $\left(t_{L}\right)$ are equal. Experimentally, efficiencies as high as $45.1 \%$ have been measured for laser and waveguide combinations with $t_{g} / t_{L}=0.34$. The tolerance to misalignment of the laser and waveguide has been theoretically and experimentally evaluated.
\end{abstract}

\section{Introduction}

An alternative to the monolithic optical integrated circuit (OIC) with all elements fabricated in a single semiconductor wafer is a hybrid OIC in which the various circuit elements (sources, detectors, modulators, etc.) are fabricated in different substrate materials and then appropriately joined together. The major advantage of the hybrid approach is that the various substrate materials can be chosen to optimize the performance of each type of device. For example, laser diode sources can be made in $\mathrm{GaAs}$, detectors in silicon, modulators in $\mathrm{LiTaO}_{3}$. However, an effective method is then needed to couple light from a waveguide in one material to one in another material. The parallel end-butt method ${ }^{1}$ is a straightforward, efficient way to accomplish such coupling. The effectiveness of this method has been demonstrated by coupling GaAs laser diodes to thin film waveguides on glass substrates. Efficient coupling of an injection laser diode to a thin film optical waveguide is difficult to achieve by using prism, grating, or tapered film couplers such as those generally used with gas lasers. The reason for this is that the injection laser has a relatively uncollimated emitted beam (divergence half angle-10-20 $)$ which is incompatible with any coupler that is strongly sensitive to the angle of incidence of the light beam. The direct end-butt coupling scheme diagrammed in Fig. 1 is capable of efficiently coupling the divergent laser beam, as will be shown in the following paragraphs.

R. G. Hunsperger and A. Lee were both with the Hughes Research Laboratories, Malibu, California 90265; R. G. Hunsperger is now with University of Delaware, Electrical Engineering Department, Newark, Delaware 19711. A. Yariv is with the California Institute of Technology, Electrical Engineering Department, Pasadena, California 91109.

Received 5 August 1976.

\section{Theory}

A. Derivation of Coupling Coefficients for Parallel Butt Coupling

In what follows we will derive an expression for the fraction of power that can be coupled from a large waveguide to a smaller waveguide by butt coupling as shown in Fig. 1.

The approach consists of expanding the fields on both sides of the butt joint in terms of the respective waveguide modes and requiring continuity across the interface of the tangential electric and magnetic field components. The analysis will be carried out for a TE wave. The results are expected to hold also for TM waves if operation is well above the propagation cutoff condition. Since we are interested in power exchange between modes the problem of mode normalization requires careful attention. A general formulation of these modes is unnecessarily complicated for our purpose. In the case of propagation well above cutoff, we can take the $y$ component of the fundamental modes as

$$
\begin{aligned}
& \mathscr{E}_{g}(x)=2\left(\frac{\omega \mu_{0}}{\beta_{g} t_{g}}\right)^{1 / 2} \cos \frac{\pi x}{t_{g}}, \\
& \mathscr{E}_{L}(x)=2\left(\frac{\omega \mu_{0}}{\beta_{L} t_{L}}\right)^{1 / 2} \cos \frac{\pi x}{t_{L}},
\end{aligned}
$$

where $\beta$ is the propagation constant, $\omega$ is the frequency, $\mu_{0}$ is the permeability, and where the $g$ and $L$ subscripts refer, respectively, to the waveguide and laser with heights $t_{g}$ and $t_{L}$ as shown in Fig. 1. In the case of the output waveguide we will also need the form of the higher order modes which may be excited. These are $^{2}$

$$
\begin{aligned}
& \mathscr{E}_{g S}(x)=2\left(\frac{\omega \mu_{0}}{\beta_{g S} t_{g}}\right)^{1 / 2} \cos \frac{\pi x S}{t_{g}}, S=1,3,5 \ldots, \\
& \mathscr{E}_{g S}(x)=2\left(\frac{\omega \mu_{0}}{\beta_{g S} t_{g}}\right)^{1 / 2} \sin \frac{\pi x S}{t_{g}}, S=2,4 \ldots
\end{aligned}
$$




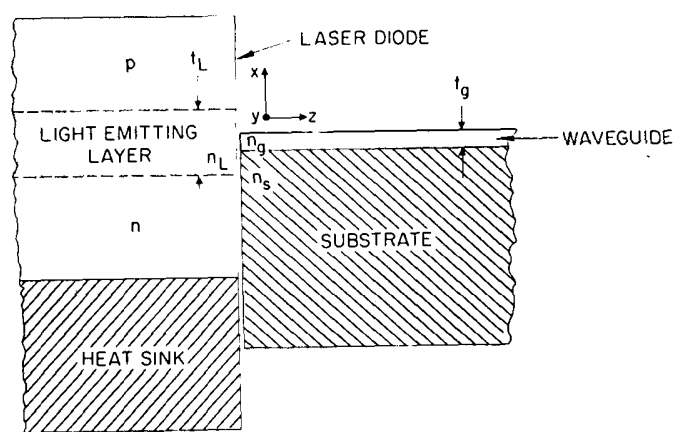

Fig. 1. Butt coupled laser colinear with waveguide.

The normalization constants of Eq. (2) are such that the integral

$$
-\frac{1}{2} \int_{-\infty}^{\infty} E_{y} H_{x} * d x=1
$$

if the fields

$$
E_{y} \text { and } H_{x}=-\frac{i}{\omega \mu_{0}} \frac{\delta \mathscr{E}_{y}}{\delta z}
$$

are taken as the mode fields as given by Eq. (2). Since

$$
-\frac{1}{2} \int_{-\infty}^{\infty} E_{y} H_{x} * d x
$$

corresponds to the power carried by the mode for unit width in the $y$ direction, it follows that a mode with a field $E_{y}=A \mathscr{E}_{g S}(x)$ carries an amount of power equal to $|A|^{2} \mathrm{~W} / \mathrm{m}$. Returning to the problem at hand, let us assume that field is incident from the left on the interface $z=0$ of Fig. 1 .

The field at $z<0$ will be taken as that of the incident fundamental mode plus a summation over the reflected modes and will be denoted as $\overline{\mathscr{E}}_{y}(x)$ :

$$
\begin{aligned}
\overline{\mathscr{E}}_{y}(x)=\overline{\mathscr{E}}_{o}(x) \exp \left(-i \bar{\beta}_{o} z\right)+r_{o} \overline{\mathscr{E}}_{o}(x) & \exp \left(-i \bar{\beta}_{o} z\right) \\
& +\sum_{k \neq 0} r_{k} \overline{\mathscr{E}}_{k}(x) \exp \left(i \bar{\beta}_{k} z\right) .
\end{aligned}
$$

The incident field $\overline{\mathscr{E}}_{o}(x)$ is taken with a unity amplitude. The reflection coefficient into mode $\overline{\mathscr{E}}_{k}$ is $r_{k}$. The incident magnetic field at $z<0$ is obtained from

$$
\begin{aligned}
\overline{\mathscr{H}}_{y}(x)=-\frac{\mathrm{i}}{\omega \mu_{o}} \frac{\delta}{\delta_{z}} \overline{\mathscr{E}}_{y}(x) & \\
=-\frac{\bar{\beta}_{o}}{\omega \mu_{o}} \overline{\mathscr{E}}_{o}(x) & \exp \left(-i \bar{\beta}_{o} z\right)+\frac{1}{\omega \mu_{o}} \sum_{k \neq 0} r_{k} \bar{\beta}_{k} \overline{\mathscr{E}}_{k}(x) \\
& \quad \times \exp \left(i \bar{\beta}_{k} z\right)+\frac{r_{o} \bar{\beta}_{o}}{\omega \mu_{o}} \overline{\mathscr{E}}_{o}(x) \exp \left(i \bar{\beta}_{o} z\right) .
\end{aligned}
$$

The transmitted field to the right of the interface (i.e., at $z>0$ ) is taken as a sum over the normal modes

$$
\begin{gathered}
\mathscr{E}_{y}(x)=\sum_{m} A_{m} \mathscr{E}_{m}(x) \exp \left(-i \beta_{m} z\right), \\
\mathscr{H}_{y}(x)=\sum_{m}-\frac{\beta_{m} A_{m}}{\omega \mu_{o}} \mathscr{E}_{m}(x) \exp \left(-i \beta_{m} z\right) .
\end{gathered}
$$

The continuity of $\mathscr{E}_{y}(x)$ at $z=0$ leads to

$$
\sum_{k \neq 0} r_{k} \overline{\mathscr{E}}_{k}(x)+\left(r_{o}+1\right) \overline{\mathscr{E}}_{o}(x)=\sum_{m} A_{m} \mathscr{E}_{m}(x),
$$

$$
\sum_{k \neq 0} \bar{\beta}_{k} r_{k} \overline{\mathscr{E}}_{k}(x)+\bar{\beta}_{o}\left(r_{o}-1\right) \overline{\mathscr{E}}_{o}(x)=\sum_{m}-\beta_{m} A_{m} \mathscr{E}_{m}(x) .
$$

We multiply, in turn, Eqs. (6) and (7) by $\mathscr{E}_{S}(x)$ and integrate over the cross section using the orthonormality condition $^{2}$

$$
\langle m / S\rangle \equiv \int_{-\infty}^{\infty} \mathscr{E}_{m}(x) \mathscr{E}_{S}(x)=\frac{2 \omega \mu_{0}}{\beta_{S}} \delta_{S, m} .
$$

The result is

$$
\begin{gathered}
\sum_{k \neq 0} r_{k}\langle\bar{k} \mid S\rangle+\left(r_{o}+1\right)\langle\overline{0} \mid S\rangle=\frac{2 \omega \mu_{o}}{\beta_{S}} A_{S}, \\
\sum_{k \neq 0} r_{k} \bar{\beta}_{k}\langle\bar{k} \mid S\rangle+\bar{\beta}_{o}\left(r_{o}-1\right)\langle\overline{0} \mid S\rangle=-2 \omega \mu_{o} \mathrm{~A}_{\mathrm{s}},
\end{gathered}
$$

where it will be recalled we are using the top bar to indicate the mode function at $z<0$, and

$$
\langle\bar{k} \mid S\rangle \equiv \int_{-\infty}^{\infty} \overline{\mathscr{E}}_{k}(x) \mathscr{E}_{S}(x) d x .
$$

Our prime interest is in solving for $\left|A_{S}\right|^{2}$, the fraction of the power coupled from the fundamental mode incident from the left $(z<0)$ into the waveguide mode to the right $(z>0)$. The unknowns appearing in Eqs. (8) and (9) are $r_{k}$ and $A_{S}$. The equations cannot be solved in their present form since the number of variables is too large. A solution for $A_{S}$ can be obtained if we assume that $\bar{\beta}_{k}=\bar{\beta}_{o}$ for all $k$, i.e., neglect the mode dispersion. This is nearly true for modes well above the propagation cutoff where $\bar{\beta}_{k} \cong(\omega / c) n_{L}$. With this assumption we rewrite Eq. (9) as

$$
\sum_{k \neq 0} r_{k}\langle\bar{k} \mid S\rangle+\left(r_{o}-1\right)\langle\overline{0} \mid S\rangle=-\frac{2 \omega \mu_{o} A_{S}}{\bar{\beta}_{o}} .
$$

Subtracting Eq. (11) from Eq. (8) gives

$$
\langle\overline{0} \mid S\rangle=\omega \mu_{0} A_{S}\left(\frac{1}{\beta_{S}}+\frac{1}{\bar{\beta}_{o}}\right) .
$$

Solving Eq. (12) for $A_{S}$ and using the definition in Eq. (7a) yield

$$
A_{S}=\frac{1}{\omega \mu_{o}}\left(\frac{\bar{\beta}_{o} \beta_{S}}{\bar{\beta}_{o}+\beta_{S}}\right) \int_{-\infty}^{\infty} \overline{\mathscr{E}}_{o}(x) \mathscr{E}_{S}(x) d x .
$$

The coupling coefficient into mode $S$ is given by $\left|A_{S}\right|^{2}$. To evaluate it we need to know the mode functions $\underline{\mathscr{E}}_{o}(x)$ and $\mathscr{E}_{S}(x)$ as well as their propagation constants $\bar{\beta}_{o}$ and $\beta_{S}$. In the limit of well confined modes we use Eq. (2) to write Eq. (13) as

$$
A_{S}=\frac{4 \bar{\beta}_{o}^{1 / 2} \beta_{S}^{1 / 2}}{\left(\bar{\beta}_{o}+\beta_{S}\right)\left(t_{g} t_{L}\right)^{1 / 2}} \int_{-t_{g} / 2}^{t_{g} / 2} \cos \frac{\pi x S}{t_{g}} \cos \frac{\pi x}{t_{L}} d x,
$$

using $\bar{\beta}_{o} \approx(\omega / c) n_{L}, \beta_{S} \approx(\omega / c) n_{S}$ in the prefactor of Eq. (14) we obtain

$$
\begin{gathered}
A_{S} \approx \frac{4\left(n_{L} n_{S}\right)^{1 / 2}}{\left(n_{L}+n_{S}\right)\left(t_{g} t_{L}\right)^{1 / 2}} \int_{-t_{g} / 2}^{t_{g} / 2} \cos \frac{\pi x S}{t_{g}} \cos \frac{\pi x}{t_{L}} d x \quad S=1,3,5 \ldots \\
A_{S} \cong 0 \quad S=2,4 \ldots
\end{gathered}
$$

Carrying out the integration yields

$$
A_{S}=\frac{8\left(n_{L} n_{S}\right)^{1 / 2}}{\pi S\left(n_{L}+n_{S}\right)} \cos \left(\frac{\pi t_{g}}{2 t_{L}}\right)\left(\frac{t_{g}}{t_{L}}\right)^{1 / 2} \frac{1}{1-\left(t_{\mathrm{g}} / S t_{L}\right)^{2}} .
$$

The fraction of the power coupled from the fundamental mode $\overline{\mathscr{E}}_{o}(x)$ at $z<0$ into the mode $S$ at $z>0$ is 


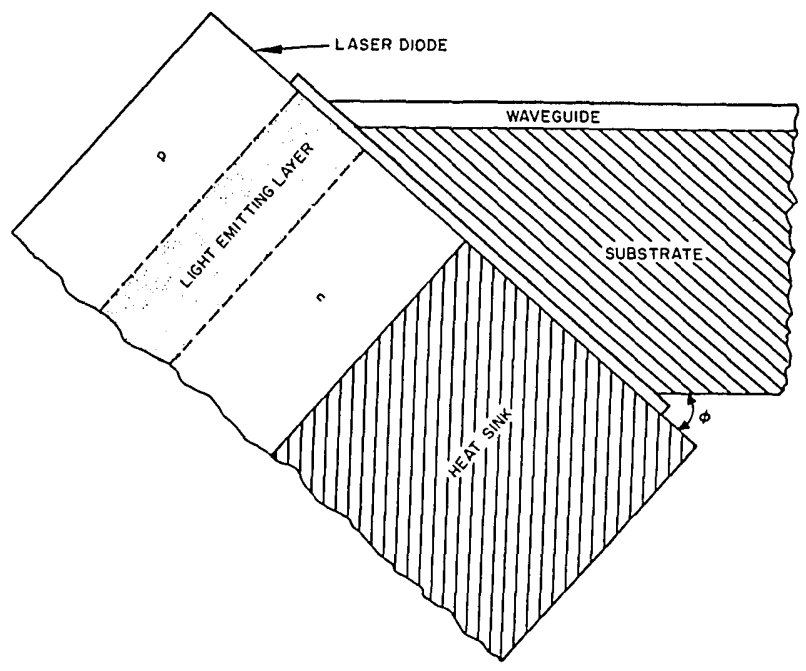

Fig. 2. Butt coupled laser angled with waveguide.

$$
\begin{gathered}
\left|A_{S}\right|^{2}=\left(\frac{8}{\pi S}\right)^{2} \frac{n_{L} n_{S}}{\left(n_{L}+n_{S}\right)^{2}} \cos ^{2}\left(\frac{\pi t_{k^{\prime}}}{2 t_{L}}\right) \\
\times \frac{1}{\left[1-\left(t_{k} / S t_{L}\right)^{2}\right]^{2}}\left(\frac{t_{g}}{t_{L}}\right) \quad S=1,3,5 \ldots \\
\left|A_{S}\right|^{2} \cong 0 \quad S=2,4 \ldots
\end{gathered}
$$

For waveguide modes far above cutoff little error is introduced by taking $n_{S} \simeq n_{g}$, and also the disappearance of the even order modes can be concisely stated by including a factor $\sin ^{2} s \pi / 2$.

$$
\begin{gathered}
\left|A_{S}\right|^{2}=\frac{64}{S^{2} \pi^{2}} \cdot \frac{n_{L} n_{k}}{\left(n_{L}+n_{k}\right)^{2}} \cdot \cos ^{2}\left(\frac{\pi t_{k}}{2 t_{L}}\right) \cdot \frac{1}{\left[1-\left(t_{k} / S t_{L}\right)^{2}\right]^{2}} \\
\cdot \frac{t_{g}}{t_{L}} \sin ^{2}\left(\frac{S \pi}{2}\right) .
\end{gathered}
$$

A comparison of calculated values for $\left|A_{S}\right|^{2}$ with experimentally measured results is given by Sec. IV.

\section{B. Angle Butt Coupling}

Here we consider the effect on the coupling coefficient $\left|A_{S}\right|^{2}$ of having the two waveguides tilted by an angle $\phi$ as in Fig. 2. Such a situation may arise if the butting planes are not exactly normal to the waveguide axes. Using the coordinate transformation

$$
\begin{aligned}
& z^{\prime}=z \cos \phi-x \sin \phi, \\
& x^{\prime}=z \sin \phi+x \cos \phi,
\end{aligned}
$$

now on the butting plane $z^{\prime}=0$ so from Eq. (16) we have $z=x \tan \phi$. The result of this transformation is to change the overlap integral in Eq. (13) to

$$
\int_{-t_{g} / 2}^{t_{g} / 2} \overline{\mathscr{E}}_{o} \frac{x}{\cos \phi} \mathscr{E}_{S}(x) \exp \left(i \beta_{S} x \tan \phi\right)_{d x} .
$$

This integral is always smaller than its counterpart in Eq. (13). The difference, however, is not appreciable until $\left(\beta_{S} t_{g} / 2\right) \tan \phi \approx \pi$ which can be written as

$$
(\tan \phi)_{\max } \approx\left(\lambda_{o}\right) /\left(n_{g} t_{g}\right),
$$

where $\lambda_{o}$ is the vacuum wavelength.

Equation (18) can be used to estimate the limiting angle at which we may expect appreciable decrease of coupling efficiency. As an example for $n_{g} \approx 2, \lambda_{o}=1$ $\mu \mathrm{m}, t_{g}=2 \mu \mathrm{m}$, we have

$$
\begin{gathered}
(\tan \phi)_{\max } \approx 1 / 4, \\
\phi_{\max } \sim 14^{\circ} .
\end{gathered}
$$

Thus we see that angle butt coupling has a lower coupling efficiency than parallel butt coupling for all angles and is not a preferred coupling technique. However, the tolerance to angular misalignment is such that a minimal decrease in coupling efficiency occurs for angles less than $\simeq 14^{\circ}$ when the parallel butt coupling technique is used for waveguides with $t_{g}=2 \mu \mathrm{m}$ and $n_{g}=2$.

\section{Experiment}

To evaluate experimentally the parallel end-butt coupling method we coupled commercially available $\mathrm{GaAs}$ laser diodes to thin film planar waveguides consisting of a sputtered layer of $\mathrm{Ta}_{2} \mathrm{O}_{5}$ on a glass (microscope slide) substrate. Single heterojunction, pulsed laser diodes were used, emitting peak pulse power up to $16 \mathrm{~W}$ at a wavelength of $9040 \AA$. The only special features of these lasers required to permit butt coupling were that the usual cap and lens were omitted, and the diodes were mounted with the light emitting cleaved endface flush with the edge of the heatsink rather than being set back slightly as is usually done.

The sputtered $\mathrm{Ta}_{2} \mathrm{O}_{5}$ waveguides used in the coupling experiments had thicknesses ranging from $1 \mu \mathrm{m}$ to $2 \mu \mathrm{m}$. To prepare the waveguides for butt coupling they were scribed and broken to produce samples of various lengths, and the end faces were dressed to $90^{\circ}$ and polished. The polishing was accomplished by a tedius but effective hand lapping and finishing process. . Alumina abrasive was used for rough lapping followed by fine polishing with cerium oxide. To prevent rounding of the critical waveguide edge, the sample to be polished was sandwiched between microscope slides waxed together. A lapping fixture was used to hold the waveguide sample perpendicular to the polishing plate containing the abrasive slurry. During polishing, samples were repeatedly examined by $1000 \times$ optical microscopy to evaluate the condition of the polished surface. After polishing they were examined in the scanning electron microscope to check the final polish to determine waveguide thickness. Under 5000× SEM examination it was possible to locate regions where no significant defects were visible over lateral lengths of $100 \mu \mathrm{m}$. From lower power optical observations we estimate that, in most samples, glitches in the surface polish took up less than $10 \%$ of the total area of the waveguide face.

The critical alignment of laser and waveguide was accomplished in the following manner. The laser and its heatsink were mounted on a piezoelectrically driven $X-Y-Z$ translation stage with an adjustment resolution 
of $40 \AA / \mathrm{V}$. The waveguide was clamped in a support structure and mounted on a stage providing tilt and rotation adjustments. Thus all the degrees of freedom needed for alignment of the relative positions of laser and waveguide were available.

\section{Results}

\section{A. Butt Coupling Power and Efficiency}

The alignment fixtures described in Sec. III were used to butt couple different combinations of lasers and waveguides. In all cases a calibrated Si photodiode was used to measure the peak output power of the laser and the peak output power emitted from the end of the waveguide when coupled to the laser. The over-all transmission efficiency (including lumped waveguide loss and input/output coupling loss) could thus be determined. Rough alignment was performed while observing the image of the waveguided light focused onto an image upconverter tube by means of a microscope objective. Final alignment was done by adjusting for maximum measured power. In addition to the measurement of overall-transmitted power we also determined the modal distribution of the power in the waveguide for most samples. This was done by clamping a strontium titanate prism to the waveguide to act as an output coupler. Light was thus coupled out of the waveguides in a pattern of $m$-lines at different angles corresponding to the $S=1,3$, and (in some cases) $S=5$ modes. The $m$-lines were clearly visible when viewed with a snooperscope on a ground glass screen placed several centimeters from the prism, and the relative power in each mode could be measured with the calibrated Si photodiode. The coupling efficiency of the prism was between about $10 \%$ and $40 \%$ and varied depending on clamping pressure and quality of surface contact. Thus only the relative power in each mode could be measured rather than the absolute power. The power in each mode and the coupling efficiency into each mode were then determined by assuming that the total power transmitted by the waveguide (as measured with the calibrated Si detector) was divided between the various modes in the same proportions as the relative powers measured in the $m$-lines. The validity of this assumption was supported by the fact that the relative proportions of power in each mode remained constant when the prism was moved to various positions along the waveguide and was always repeatable even though the absolute power levels measured varied considerably, depending on prism clamping pressure, etc. The constancy of these ratios also indicates that intermode coupling or mode conversion was not occurring as the light traveled along the waveguide. Since all the modes observed in these waveguides were well confined, and far from cutoff we also assumed that the prism coupling efficiency was the same for all modes. Any error introduced by this assumption would tend to make the resulting calculated power in the lowest order mode appear less than actual, and the values for higher order modes appear greater than actual. This is because the lowest order mode is theoretically better confined than the higher order ones (which are closer to cutoff), and therefore the prism coupling efficiency should be worst for the lowest order mode. Thus the values for power coupled into the lowest order mode that we have determined are conservative.

In the course of perfecting the alignment techniques, a great many measurements of total power coupled and modal distribution were made. However the significant data can be concisely summarized as shown in Table I where only the best results achieved for each waveguide are tabulated, since these represent the closest we were able to come to ideal alignment in each case. It should be noted that the efficiencies shown in Table I are actually over-all transmission efficiencies rather than input coupling efficiencies since they have not been corrected for either waveguide attenuation or for reflection loss at the output of the waveguide.

Looking at these raw data one can see the significant effect of waveguide attenuation in that the efficiencies for long waveguides are much less than those for short ones. From the dependence of measured efficiency on waveguide length, we estimate the waveguide attenuation to be approximately $2 \mathrm{~dB} / \mathrm{cm}$. In the case of waveguide DC-2 detailed measurements were made of the attenuation loss. A laser diode was coupled to the waveguide and clamped in position to maintain constant input coupling. Then the output coupling prism was moved to various positions along the length of the waveguide, and the relative power in each mode was measured as previously described. There was a significant scatter in the data, probably because of variations in the prism coupling efficiency from one spot to another. Nevertheless we were able to determine loss coefficients by drawing a straight line through the points on a semilog plot of relative power vs distance. The results indicated the following losses for the three modes observed:

$$
\begin{array}{ll}
S=1 & 1.6 \mathrm{~dB} / \mathrm{cm} ; \\
S=3 & 1.6 \mathrm{~dB} / \mathrm{cm} ; \\
S=5 & 1.8 \mathrm{~dB} / \mathrm{cm} .
\end{array}
$$

The reflection loss at a $\mathrm{Ta}_{2} \mathrm{O}_{5}$ waveguide output to air is

$$
R=\left(\frac{n_{2}-n_{1}}{n_{2}+n_{1}}\right)^{2}=\left(\frac{2.0-1}{2.0+1}\right)^{2}=0.11 .
$$

When the measured efficiency data of Table I for $\mathrm{Ta}_{2} \mathrm{O}_{5}$ waveguides are corrected for the above output reflection losses and also for an assumed waveguide attenuation of $1.6 \mathrm{~dB} / \mathrm{cm}$ for waveguide DC-2 and 2 $\mathrm{dB} / \mathrm{cm}$ for the others, the values shown in Table II result. These values probably more accurately represent the actual input coupling efficiencies achieved than do the uncorrected data of Table I.

In Sec. II a theoretical expression was derived for the coupling coefficient $\left|A_{s}\right|^{2}$ for the case of parallel end butt coupling [Eq. (15)]. Figure 3 shows the normalized curves of $\left|A_{s}\right|^{2}$ vs the ratio of waveguide thickness $\left(t_{g}\right)$ to laser light emitting layer thickness $\left(t_{L}\right)$. The solid curves are the theoretical curves for the three lowest order TE modes in the case of $t_{L}=5.8 \mu \mathrm{m}$. The specific choice of $5.8 \mu \mathrm{m}$ rather than the value of $t_{L} \simeq 5 \mu \mathrm{m}$ 
Table I. Measured Coupling Efficiencies Based on Transmitted Power Uncorrected for Waveguide Losses

\begin{tabular}{|c|c|c|c|c|c|c|c|c|c|}
\hline \multirow{3}{*}{$\begin{array}{c}\text { Label } \\
\begin{array}{c}\text { Laser } \\
\text { wave- } \\
\text { guide }\end{array}\end{array}$} & \multicolumn{3}{|c|}{ Waveguide } & \multirow{3}{*}{$\begin{array}{l}\text { Laser } \\
\text { emitted } \\
\text { power } \\
\text { W }\end{array}$} & \multirow{3}{*}{$\begin{array}{c}\text { Wave- } \\
\text { guide } \\
\text { trans- } \\
\text { mitted } \\
\text { power } \\
\text { W }\end{array}$} & \multirow{2}{*}{\multicolumn{4}{|c|}{$\begin{array}{c}\text { Efficiency } \\
\text { (percent of laser emitted power) }\end{array}$}} \\
\hline & \multirow[b]{2}{*}{ Material } & \multirow{2}{*}{$\begin{array}{c}\text { Thick- } \\
\text { ness } \\
\mu \mathrm{m}\end{array}$} & \multirow{2}{*}{$\begin{array}{c}\text { Length } \\
\mathrm{cm}\end{array}$} & & & & & & \\
\hline & & & & & & Total & $\begin{array}{l}\text { Mode } \\
s=1\end{array}$ & $\begin{array}{l}\text { Mode } \\
s=3\end{array}$ & $\begin{array}{l}\text { Mode } \\
s=5\end{array}$ \\
\hline $\begin{array}{c}\text { LD67-8 } \\
\text { DC-2 }\end{array}$ & $\mathrm{Ta}_{2} \mathrm{O}_{5}$ & 1.0 & 4.0 & 12.8 & 0.65 & 5.0 & 4.0 & 0.81 & 0.19 \\
\hline $\begin{array}{c}\text { LD69-1 } \\
\text { RF-1 }\end{array}$ & $\mathrm{Ta}_{2} \mathrm{O}_{5}$ & 1.7 & 4.0 & 16 & 0.84 & 5.2 & 4.4 & 0.8 & - \\
\hline $\begin{array}{c}\text { LD67-3 } \\
\text { DC-1 }\end{array}$ & $\mathrm{Ta}_{2} \mathrm{O}_{5}$ & 2.0 & 4.0 & 16 & 1.04 & 6.5 & 5.2 & 1.3 & - \\
\hline $\begin{array}{c}\text { LD67-7 } \\
\text { DC-1 }\end{array}$ & $\mathrm{Ta}_{2} \mathrm{O}_{5}$ & 2.0 & 1.9 & 12.8 & 2.39 & 19 & 17.0 & 2.0 & - \\
\hline $\begin{array}{c}\text { LD69-2 } \\
\text { RF-1 }\end{array}$ & $\mathrm{Ta}_{2} \mathrm{O}_{5}$ & 1.7 & 0.7 & 2.08 & 0.56 & 27 & & & \\
\hline $\begin{array}{c}\text { LD69-1 } \\
\text { DC-1 }\end{array}$ & $\mathrm{Ta}_{2} \mathrm{O}_{5}$ & 2.0 & 0.7 & 16 & 8.32 & 52 & & & \\
\hline
\end{tabular}

Table II. Coupling Efficiencies Corrected for Waveguide Attenuation and Output Reflection Loss

\begin{tabular}{|c|c|c|c|c|c|c|c|c|c|}
\hline \multirow{3}{*}{$\begin{array}{l}\text { Label } \\
\text { Laser } \\
\text { wave- } \\
\text { guide }\end{array}$} & \multicolumn{3}{|c|}{ Waveguide } & \multirow{3}{*}{$\begin{array}{c}\text { Laser } \\
\text { emitted } \\
\text { power } \\
\text { W }\end{array}$} & \multirow{3}{*}{$\begin{array}{c}\text { Coupled } \\
\text { wave- } \\
\text { guide } \\
\text { power } \\
\text { W }\end{array}$} & \multirow{2}{*}{\multicolumn{4}{|c|}{$\begin{array}{c}\text { Coupling efficiency } \\
\text { (percent of laser emitted power) }\end{array}$}} \\
\hline & \multirow[b]{2}{*}{ Material } & \multirow{2}{*}{$\begin{array}{c}\text { Thick- } \\
\text { ness } \\
\mu \mathrm{m}\end{array}$} & \multirow{2}{*}{$\begin{array}{c}\text { Length } \\
\mathrm{cm}\end{array}$} & & & & & & \\
\hline & & & & & & Total & $\begin{array}{l}\text { Mode } \\
s=1\end{array}$ & $\begin{array}{l}\text { Mode } \\
s=3\end{array}$ & $\begin{array}{l}\text { Mode } \\
s=5\end{array}$ \\
\hline $\begin{array}{c}\text { LD67-8 } \\
\text { DC-2 }\end{array}$ & $\mathrm{Ta}_{2} \mathrm{O}_{5}$ & 1.0 & 4.0 & 12.8 & 3.19 & 24.9 & 20.0 & 4.0 & 0.9 \\
\hline $\begin{array}{c}\text { LD69-1 } \\
\text { RF-1 }\end{array}$ & $\mathrm{Ta}_{2} \mathrm{O}_{5}$ & 1.7 & 4.0 & 16 & 5.94 & 37.1 & 31.3 & 5.8 & - \\
\hline $\begin{array}{c}\text { LD67-3 } \\
\text { DC-1 }\end{array}$ & $\mathrm{Ta}_{2} \mathrm{O}_{5}$ & 2.0 & 4.0 & 16 & 7.36 & 46.1 & 36.9 & 9.2 & - \\
\hline $\begin{array}{c}\text { LD67-7 } \\
\text { DC-1 }\end{array}$ & $\mathrm{Ta}_{2} \mathrm{O}_{5}$ & 2.0 & 1.9 & 12.8 & 6.44 & 50.3 & 45.1 & 5.2 & - \\
\hline $\begin{array}{c}\mathrm{LD} 69-2 \\
\mathrm{RF}-1\end{array}$ & $\mathrm{Ta}_{2} \mathrm{O}_{5}$ & 1.7 & 0.7 & 2.08 & 0.87 & 41.6 & & & \\
\hline $\begin{array}{l}\text { LD69-1 } \\
\text { DC-1 }\end{array}$ & $\mathrm{Ta}_{2} \mathrm{O}_{5}$ & 2.0 & 0.7 & 16 & 12.9 & 80.6 & & & \\
\hline
\end{tabular}

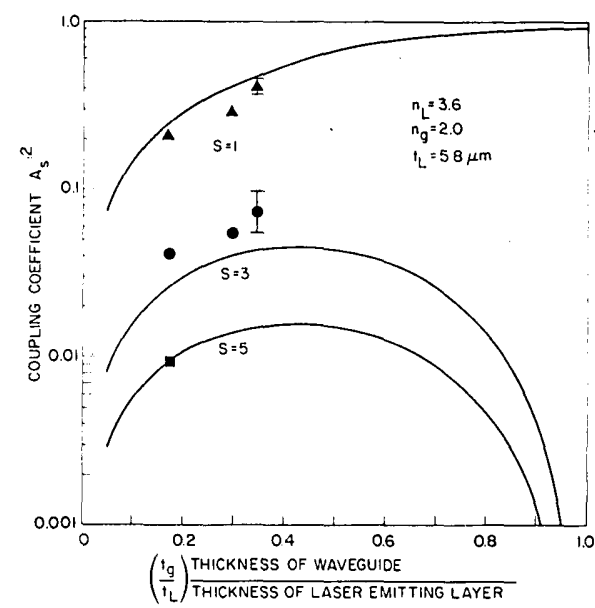

Fig. 3. Comparison of theoretical and experimental coupling coefficient data for $t_{L}=5.8 \mu \mathrm{m}$.

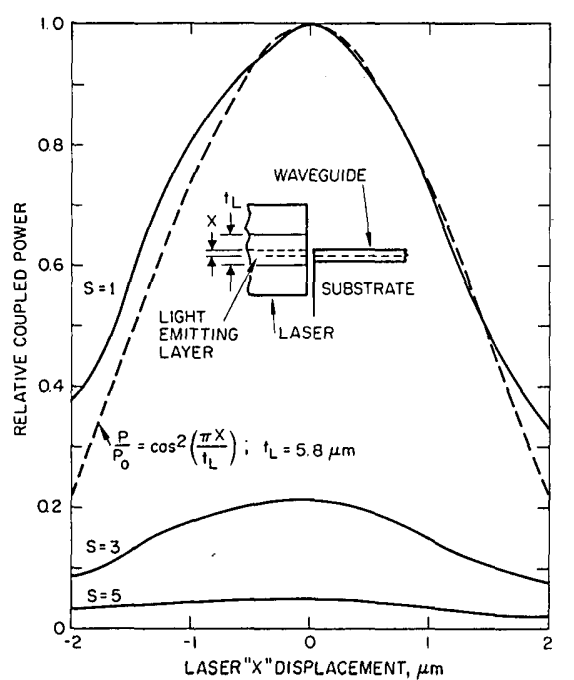

Fig. 4. Tolerance to transverse displacement. 
determined from near field pattern measurements is based on an analysis of tolerance to lateral transverse misalignment of the laser and waveguide as discussed in the next paragraph. The plotted points are the experimentally determined values of coupling coefficients from Table II.

\section{B. Tolerances}

The tolerance to transverse displacement of the laser relative to the waveguide was experimentally studied by butt coupling laser LD-67-8 to waveguide DC-2 with the alignment fixtures, then intentionally moving the laser laterally in the transverse $(x)$ direction by changing the voltage on the piezoelectric micrometer. Because of the high resolution of the PZM micrometer $(40 \AA / \mathrm{V})$ an accurate curve of relative coupled power vs laser displacement was obtainable. The results are shown in Fig. 4. The solid curves are the experimentally measured coupled power data for the three lowest order modes, while the dotted curve is the theoretically expected coupled power variation for the fundamental mode assuming a laser light emitting layer thickness $t_{L}$ $=5.8 \mu \mathrm{m}$. This value of thickness was chosen to fit the experimental data to the theoretical curve for laser displacements toward the surface $(+x)$ of the waveguide. The broadening of the experimental curve for laser displacements toward the substrate $(-x)$ results because the step in the index of refraction profile is not as great as the waveguide-substrate interface as it is at the waveguide-air interface. Thus the waveguide mode is not as well confined on the substrate side. This effect is even more noticeable for the higher order modes, which are somewhat closer to cutoff and hence less well confined.

While the coupling efficiency was found to be very sensitive to transverse misalignment the most sensitive parameter was found to be the spacing between laser and waveguide. The spacing tolerance data of Fig. 5 for laser LD-67-8 and waveguide DC-2 illustrate why that was so. The coupled power decreased when laser to waveguide spacing was increased, remaining relatively large over a distancelof several wavelengths as one would expect. However the decrease was not uniform. The magnitude of the coupled power oscillated between peaks (traced by the solid curve) and minima (traced by the dotted curve). The spacing between the peaks was equal to $\frac{1}{2}$ wavelength. This effect resulted from the fact that the polished end face of the laser and the polished end face of the waveguide together formed a Fabry-Perot interferometer which acted to modify the effective reflectance of the laser output face, thereby altering the coupled power. The practical significance of this observation is that the resolution of the adjustment to laser-waveguide spacing must be better than $0.1 \mu \mathrm{m}$ to enable one to obtain peak coupling efficiency, even though the total spacing can be as much as $4 \mu \mathrm{m}$ without causing more than a factor of 0.5 decrease in coupled power. The Fabry-Perot interferometer effect could in principle be eliminated by using an index matching fluid or epoxy between the laser and waveguide. However the practical difficulties in achieving

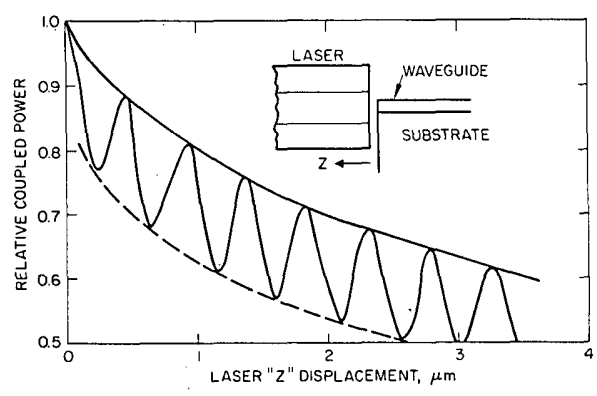

Fig. 5. Tolerance to laser/waveguide spacing.

a stable match appear to be prohibitive for the limited improvement in coupling that would result.

\section{v. Conclusions}

The work described in this paper has established that the parallel end butt coupling method is a viable technique for coupling an injection laser to a thin film waveguide. Theoretical calculations predict that most of the optical power is coupled into the lowest order mode, and that coupling efficiencies into that mode as high as $90 \%$ can be achieved for the case of a GaAs laser and $\mathrm{Ta}_{2} \mathrm{O}_{5}$ waveguide both with the same thickness light emitting or guiding layer. As the thickness of the waveguide is decreased relative to that of the laser the coupling efficiency into the lowest order mode is decreased, and more power is coupled into the higher order modes. However, even for a ratio of $t_{g} / t_{L}=0.5$ the theoretical maximum coupling into the lowest order mode is $60 \%$, while that into the next higher $(S=3)$ mode is $4 \%$. The theory also predicts that angling the end face of the waveguide to increase effective area does not improve the coupling efficiency because the competing reduction in coupling resulting from misalignment of the optical fields in the laser and waveguide is dominant. Thus the parallel end butt coupling colinear with the waveguide is best. The experimental results achieved agree well with the theoretical predictions. Over-all transmission efficiencies determined by measuring power emitted from the waveguide were as high as $52 \%$ for the case of $2.0-\mu \mathrm{m}$ thick waveguide. When this value was corrected for waveguide attenuation and output reflection loss the calculated input coupling efficiency was $80 \%$. Measurements of modal power distribution confirmed that more than $80 \%$ of the power coupled into the waveguide went into the lowest order mode in all the cases examined. Only the $S=1,3$, and in some cases $S=5$ modes were observable in the waveguides, as predicted by the theory.

In order to achieve the coupling efficiencies that have been reported, critical alignment of the laser and waveguide necessitates the use of piezoelectrically driven fixtures or some equivalent means. The spacing between the laser and waveguide and the transverse lateral waveguide-laser alignment are the most critical parameters, requiring an adjustment resolution of better than $0.1 \mu \mathrm{m}$ to ensure optimum coupling. $\mathrm{Pi}$ ezoelectric micrometers with resolution of $40 \AA / \mathrm{V}$ were 
effective in this application. The various tilt and rotational alignment parameters were found to be much less critical and could be adjusted for optimum coupling with a relatively unsophisticated mechanical stage.

Ultimately, it should be possible to obtain even better coupling efficiency with a cw laser than with the pulsed laser diodes which we used. This is true because the $\mathrm{cw}$ lasers are double heterojunction diodes with light confinement on both sides of the P-N junction to an emitting layer thickness of $0.25 \mu \mathrm{m}$. Compared to the $5-\mu \mathrm{m}$ emitting layer thickness of pulsed diode lasers, this offers a potential advantage of a factor of 20 in increased $t_{g} / t_{L}$ ratio. However, increased beam divergence due to diffraction in the cw laser makes close spacing of the laser and waveguide absolutely imperative. The need for the laser to be flush mounted or protruding on the heatsink presents a particular problem in the case of present day cw lasers which require extremely good heatsinking. As improvements in laser and heatsink design are made, this problem will become less significant.

This work was supported by the Air Force Avionics Laboratory, Air Force Systems Command, United States Air Force, Wright-Patterson AFB, Ohio, under contract F33615-75-C-1024.

\section{References}

1. R. G. Hunsperger and A. Lee, in Digest of Technical Papers, OSA Topical Meeting on Integrated Optics, Salt Lake City, Utah, 12-14 January 1976.

2. A. Yariv, IEEE J. Quantum Electron. QE-9, 919 (1973).

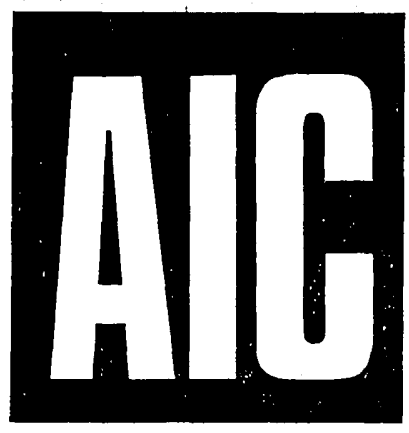

International Colour Association
Association Internationale de la Couleur
Internationale Vereinigung für die Farbe

COLOR 77TROY

Address for reply:

Fred W. Billmeyer, Jr.

Dept. of Chemistry, MRC Rm. 217

Rensselaer Polytechnic Institute

Troy. New York 12181, U.S.A.

(518) $270-6458$

SECOND CIRCULAR AND REGISTRATION FORMS

The Organizing Committee for COLOR 77 wishes

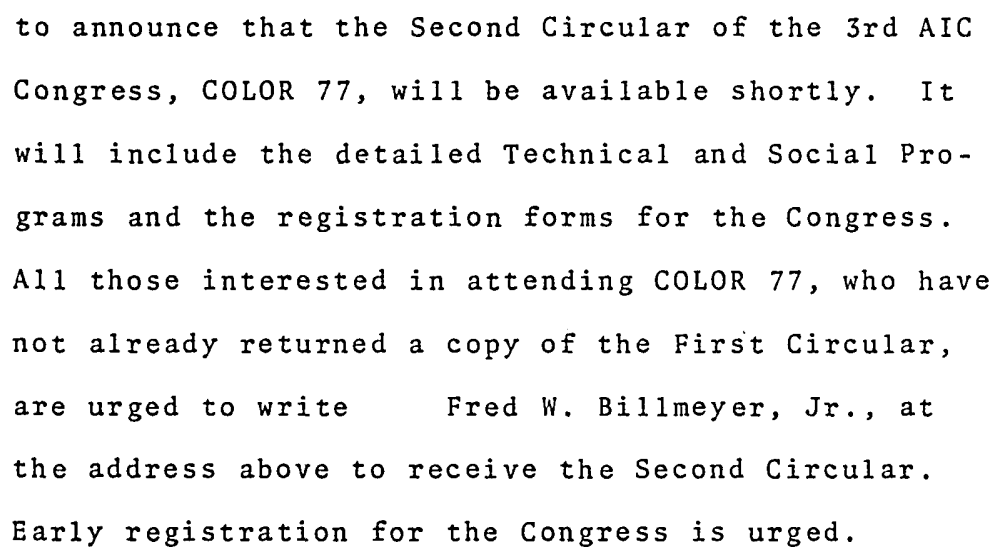

